Trauma Berufskrankh 2008 10 [Suppl 1]:133-137

Online publiziert: 18. Januar 2008

(c) Springer Medizin Verlag 2008
DOI 10.1007/s10039-007-1342-0

\author{
M. Diefenbeck ${ }^{1}$ T. Mückley ${ }^{1}$ G.O. Hofmann ${ }^{1,2}$ \\ ${ }^{1}$ Klinik für Unfall-, Hand- und Wiederherstellungschirurgie, \\ Friedrich-Schiller-Universität, Jena \\ ${ }^{2}$ Klinik für Unfall- und Wiederherstellungschirurgie, \\ BG-Kliniken Bergmannstrost, Halle, Saale
}

\section{Multiresistente Erreger im Krankenhaus}

\section{Konsequenzen für die chirurgische Behandlung}

Multiresistente Erreger (MRE) haben sich in den vergangenen Jahren zu einem zentralen Problem des Hygienemanagements im Krankenhaus entwickelt [13]. Hinzu kommt, dass multiresistente Keime zunehmend in ambulanten medizinischen Einrichtungen sowie Alten- und Pflegeheimen übertragen werden und so die Verbreitung der Keime beschleunigt wird [19].

MRE sind Krankeitserreger, die aufgrund ihrer Resistenz nur noch sehr eingeschränkt antibiotisch behandelt werden können. Mittlerweile ist für eine Vielzahl von Bakterien eine Mehrfachresistenz beschrieben, z. B. vancomycinresistente Enterokokken (VRE) [5] oder multiresistenter Pseudomonas aeruginosa [9]. Für das Hygienemanagement im Krankenhaus steht jedoch weiterhin der methicilinresistente Staphylococcus aureus (MRSA) im Vordergrund. Seiner Benennung liegt die Resistenz gegenüber Methicillin bzw. heute Oxacillin zugrunde, weshalb im klinischen Alltag MRSA und ORSA (oxacillinresistenter Staphylococcus aureus) synonym verwendet werden. Definiert wird der Keim durch das Vorhandensein des Penicillinbindeproteins $\mathrm{PBP}_{2 \mathrm{a}}$, welches durch das MECA-Gen kodiert wird. Alle $\beta$-Laktam-Antibiotika, einschließlich der Cephalosporine und Carbapeneme sind zurzeit als unwirksam gegen MRSA anzusehen [11].

Seit dem ersten Auftreten im Jahr 1961 hat die MRSA-Häufigkeit weltweit zuge- nommen. Der Anstieg der MRSA-Prävalenz in deutschen Krankenhäusern verlief im Mittel von 1990 mit 1,7\% MRSA an allen Staphylococcus-aureus-Isolaten über 8,7\% im Jahr 1995, 15,2\% im Jahr 1998 auf etwa $20 \%$ im Jahr 2001 [20]. Im Jahresbericht 2005 des „European Antimicrobial Resistance Surveillance System“" (EARSS) wurde die MRSA-Inzidenz aus invasiven Isolaten für Deutschland mit $21 \%$ angegeben (- Abb. 1).

Die Überwachung der MRSA-Ausbreitung ist dadurch erschwert, dass es oft nur $\mathrm{zu}$ einer symptomlosen Besiedelung des
Patienten und nicht zu einer manifesten Infektion kommt. Gerade aber diese Personen können zu einer Verbreitung von MRSA beitragen.

\section{Risiokofaktoren: Erreger - Patient - Implantat}

Das Problem der MRSA-Besiedelung eines Patienten besteht in der Gefahr einer nachfolgenden MRSA-Infektion. Tatsächlich entwickeln 19-25\% der MRSA-kolonisierten Patienten eine Infektion durch MRSA [2].

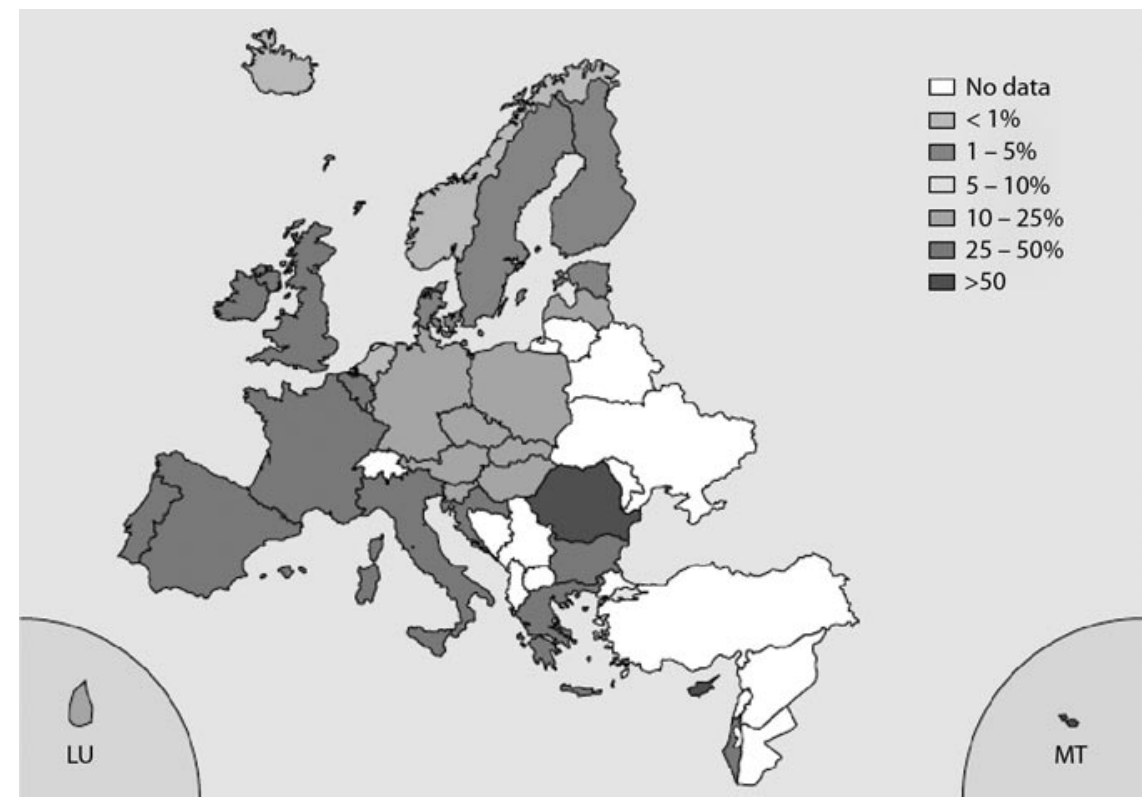

Abb. $1 \Delta$ Anteil des MRSA an allen invasiven Staphylococcus-aureus-Isolaten in Europa 2005. Aus [4]) 


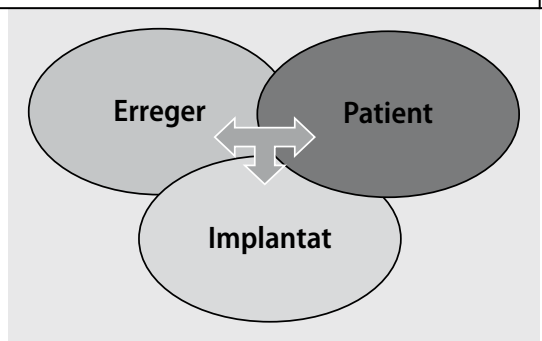

Abb. $2 \Delta$ Infektionsrisiko durch MRSA: Zusammenspiel von erreger-, patienten- und implantatabhängigen Risikofaktoren

Beispiele hierfür sind im Fachbereich der Orthopädie und Unfallchirurgie eine periprothetische MRSA-Infektion nach Implantation einer Hüfttotalendoprothese bei Koxarthrose oder eine akute MRSA-Osteitis nach operativ versorgter hüftgelenknaher Fraktur, beides im Fall eines nicht erkannten MRSA-Trägers.

Dieses Infektionsrisiko resultiert aus einem Zusammenspiel von erreger-, patienten- und implantatabhängigen Risikofaktoren (• Abb. 2).

\section{Risikofaktor Erreger}

Staphylococcus aureus kommt ubiquitär vor und gehört bei 15-40\% der Menschen zur normalen Haut- und Schleimhautflora. Er bevorzugt feuchte Körperregionen und besiedelt insbesondere die Leiste und die Nase. Aufgrund ihrer hohen Umweltresistenz können diese Bakterien über Monate in der unbelebten Umwelt überleben und nachfolgend über kontaminierte Gegenstände oder Hände auf andere Personen übertragen werden. Durch den zunehmenden, häufig unkritischen und unkontrollierten Einsatz von Breitbandantibiotika wird die Normalflora der Haut verändert, und der steigende Selektionsdruck führt zur weiteren Verbreitung multiresistenter Erreger, insbesondere des MRSA [13].

Die zentrale und viel diskutierte Frage des „Risikofaktors MRSA“ ist, ob eine manifeste Infektion mit MRSA im Vergleich zum methicillinsensiblen Staphylococcus aureus (MSSA) pathogener und aggressiver verläuft und letztendlich die Prognose für den Patienten schlechter ist.

In Publikationen aus der Gefäßchirurgie wurden Mortalitätsraten von 56$100 \%$ nach aorto-iliakalen Rekonstruktionen bzw. alloplastischem Aortenersatz mit nachfolgender MRSA-Infektion an-

\section{Multiresistente Erreger}

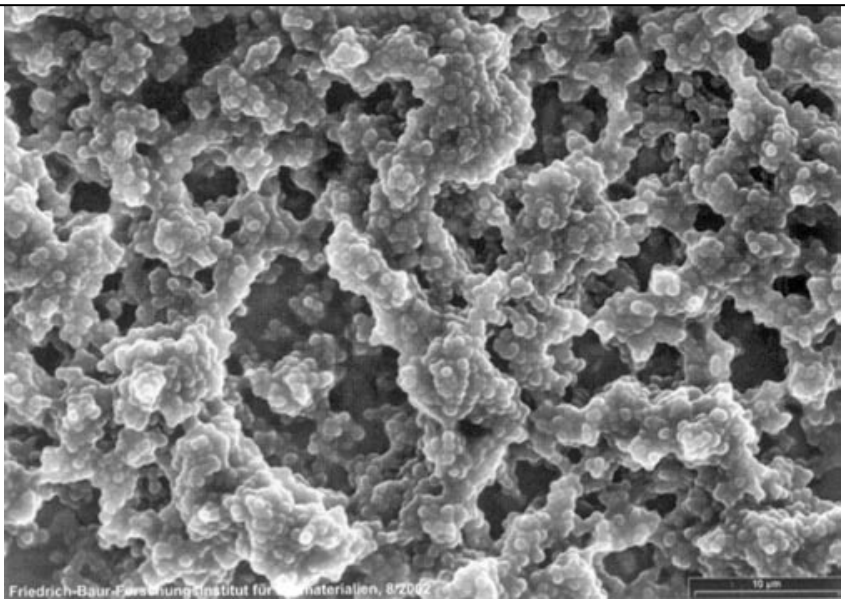

Abb. $3<\ln$ Biofilm eingebettete Staphylococcus-aureus-Kolonie (zur Verfügung gestellt von W. Mittelmeier, Technische Universität München)

gegeben $[8,15]$. Kilgus et al. [12] fanden für die Hüft- und Knieprotheseninfektionen durch MRSA deutlich schlechtere Ergebnisse im Vergleich zu Endoprotheseninfekten durch MSSA. Bei Hüftprotheseninfektionen durch sensible Bakterien konnten $81 \%$ erfolgreich behandelt werden, im Fall von MRSA-Infekten nur $48 \%$. Bei Knieprotheseninfekten war der Unterschied noch deutlicher: 89\% Erfolg bei sensiblen Erregern, nur 18\% bei resistenten Keimen [12]. Interessanterweise wurde in einer aktuellen Arbeit über den Vergleich von Gelenkinfektionen, verursacht durch MRSA vs. MSSA, kein signifikanter Unterschied in Hinblick auf Behandlungsergebnis (Outcome), Dauer der Antibiose und Auftreten von Reinfektionen gefunden [1]. Jedoch war die Mortalität nach 6 Monaten in der MRSA-Gruppe tendenziell höher. Dies wurde darauf zurückgeführt, dass in dieser Gruppe deutlich ältere Patienten (im Mittel 76 vs. 44 Jahre) mit signifikant höherer Komorbidität waren [1]. Andere Autoren kamen ebenfalls zu dem Schluss, dass die erhöhte Mortalität bei MRSA-Infekten hauptsächlich durch die Nebenerkrankungen und das erhöhte Patientenalter bedingt ist. Demgegenüber gaben 2 Studien, in denen versucht wurde, sämtliche Kofaktoren auszuschließen und nur die Infektionserreger MRSA und MSSA einander gegenüber zu stellen, eine 2,7-fache Erhöhung des relativen Risikos in Bezug auf die Mortalität bei proximalen Femurfrakturen mit nachfolgender Infektion und eine 2,12-fache Erhöhung bei MRSA-Bakteriämie an $[16,22]$.

\section{Risikofaktor Implantat}

Bei den meisten Operationen im Fachgebiet Orthopädie/Unfallchirurgie werden Implantate in Form von Osteosynthesen oder Endoprothesen eingebracht. Diese stellen zunächst einen Fremdkörper im Organismus dar. Unmittelbar nach dem Einbringen des Materials beginnt ein Wettlauf zwischen der Inkorporation des Fremdkörpers durch den Organismus und der Besiedelung durch möglicherweise anwesende Bakterien. Diesen Wettlauf nannten Gristina et al. [7] „race for the surface“. Die einzelnen Schritte sind:

- die Bildung eines konditionierenden Films auf dem Implantat,

- die Adhäsion von Zellen und Bakterien und

- die Bildung eines Biofilms

(• Abb. 3).

Folgen sind entweder die reizlose Integration des Implantats oder im anderen Extrem die periprothetische bakterielle Infektion.

Um einen Wundinfekt auszulösen, sind normalerweise etwa $10^{6}$ Bakterien nötig, bei Gewebeschäden noch $10^{4}$ und bei der Kombination von Gewebeschäden und dem Vorhandensein eines Implantats nur noch $10^{2}$ [3].

\section{Risikofaktor Patient}

In den letzten Jahren wurden viele Risikofaktoren der MRSA-Besiedelung erkannt. Dazu zählen $[11,16,22]$ :

- hohes Lebensalter,

- Vorbehandlung auf einer Intensivstation, 
- vorhergehender stationärer Krankenhausaufenthalt oder

- Aufenthalt in einem Pflegeheim.

Krankheiten, die das MRSA-Risiko steigern sind:

- Diabetes mellitus,

- Niereninsuffizienz,

- Psoriasis,

- Verbrennungen,

- periphere arterielle Verschlusskrankheit (pAVK) und

- Ulcera cruris.

Risikofaktoren durch vorhergegangene medizinische Maßnahmen sind:

- Implantate (z. B. Endoprothesen),

- Harnwegskatheter,

- i.v. Zugänge über längere Zeit,

- Dialyse,

- vorhergehende Behandlung mit einem Antibiotikum,

- Chemotherapie und

- Immunsuppression.

\section{Ökonomische Auswirkungen}

Es ist offensichtlich, dass Infektionen durch resistente Erreger zu einer längeren Verweildauer im Krankenhaus führen und höhere Kosten für die Diagnostik, Therapie und Isolierungsmaßnahmen anfallen $[6,10]$. Die finanziellen Mehraufwendungen für einen MRSA-Patienten auf einer Intensivstation belaufen sich auf 1622 EUR/Tag [6]. Die täglichen Mehrkosten auf einer orthopädischen Normalstation wurden auf 536 EUR geschätzt [14]. Zusätzlich wurden die Kosten für die Sperrung von Betten für die notwendigen Isolierungsmaßnahmen an einem Großklinikum mit 210.00o EUR/Jahr beziffert [17].

Von Nixon et al. [16] wurde 2006 eine Studie zur MRSA-Inzidenz, -Verbreitung, -Mortalität, -Kosten und -Kontrolle an einer orthopädisch/unfallchirurgischen Abteilung in England publiziert. Bei allen stationären Aufnahmen wurde von Januar 2003-Mai 2004 ein MRSAScreening durchgeführt (5594 Zugänge, 22.810 mikrobiologische Abstriche). 1,3\% der elektiven Zugänge und 3,8\% der Notfallpatienten waren mit MRSA besiedelt. Die Kosten für das MRSA-Screening und ggf. die Sanierung betrugen 83.300 £ (etwa

Trauma Berufskrankh 2008 · 10[Suppl 1]:133-137 DOI 10.1007/s10039-007-1342-0

○) Springer Medizin Verlag 2008

\section{Diefenbeck ·T. Mückley · G.O. Hofmann \\ Multiresistente Erreger im Krankenhaus. Konsequenzen für die chirurgische Behandlung}

\section{Zusammenfassung}

Multiresistente Erreger (MRE) sind Krankeitserreger, die aufgrund ihrer Resistenz nur noch sehr eingeschränkt antibiotisch behandelt werden können. Für das Hygienemanagement im Krankenhaus steht weiterhin der methicillinresistente Staphylococcus aureus (MRSA) im Vordergrund. Seine aktuelle Prävalenz beträgt in Deutschland 21\%. In Kombination mit der zunehmenden MRSA-Zirkulation zwischen verschiedenen Einrichtungen des Gesundheitssystems, der erschwerten effektiven Therapie von manifesten MRSA-Infektionen und knapper werdenden Ressourcen im Gesundheitssystem stellt dies eine kritische Situation dar. Nachdem in den letzten Jahren die Risikofaktoren für die MRSA-Besiedelung und -Übertragung erkannt worden sind, muss nun ein effektives MRSA-Management zur Bekämpfung und Eindämmung der resistenten Erreger eingesetzt werden. Eckpunkte dieser Surveillancemaßnahmen sind: primäres MRSA-Screening, krankenhaushygienische Maßnahmen, konsequente chirurgische Sanierung von Infektionen, Information der Betroffenen, ihrer Angehörigen und weiterbehandelnden Einrichtungen sowie Fort- und Weiterbildung aller an der Versorgung beteiligten Berufsgruppen.

\section{Schlüsselwörter}

Multiresistente Erreger (MRE) - Methicillinresistenter Staphylococcus aureus (MRSA) . Prävention von Infektionen .

MRSA-Management · Surveillance

\section{Multiresistant hospital pathogens. Consequences for surgical treatment}

\section{Abstract}

Infections caused by multiresistant pathogens can be treated only with a limited number of antibiotics. Methicillin-resistant Staphylococcus aureus (MRSA) remains the most important resistant bacterium in the context of hospital surveillance and prevention of nosocomial infections. The prevalence of MRSA is currently $21 \%$ in Germany. In combination with the cycle of MRSA transmission among various healthcare facilities, difficulties in effectively treating MRSA, surgical site infections, and the growing shortage of resources in the healthcare system, this is becoming a more and more critical situation. Risk factors for MRSA colonisation and transmission have been described in previous years, and an effective MRSA management program is now needed to control further increase in MRSA infections. This involves selective screening for MRSA, strict hospital hygiene, effective surgical treatment of infections, and education of patients, relatives, and all hospital staff.

\section{Keywords}

Multiresistant pathogens · Methicillinresistant Staphylococcus aureus (MRSA) . Infection prevention - MRSA management . Surveillance 


\section{Infobox}

Informationen bezüglich

Hygienemaßnahmen im Umgang mit MRE

- http://www.rki.de

http://www.awmf-online.de

- http://www.aerzteblatt.de

125.560 EUR) pro Jahr. Durch die Screening-, Isolierungs- und Dekontaminationsmaßnahmen traten im Vergleich zum Vorjahr 26 MRSA-Infektionen weniger auf. Die zusätzlichen Kosten der Behandlung einer manifesten MRSA-Infektion nach proximaler Femurfraktur [durchschnittlich 50 Tage längerer Krankennhausaufenthalt, zusätzliche 19 Tage Vancomycintherapie, zusätzliche 26 Tage VACTherapie (VAC: „vacuum-assisted-closu$\mathrm{re}^{\text {") })] ~ w u r d e n ~ g e g e n u ̈ b e r ~ e i n e r ~ I n f e k t i o n ~}$ mit MSSA mit 13.973£ (etwa 21.061 EUR) pro Patient berechnet. Bei 26 MRSA-Infektionen pro Jahr ergibt das eine Summe von $363.298 £$ (etwa 547.609 EUR) im Vergleich zu 83.300 $£$ (etwa 125.56o EUR) für Screening- und Sanierungsmaßnahmen. Diese Zahlen verdeutlichen, wie sinnvoll ein MRSA-Sceening auch aus ökonomischen Gesichtspunkten ist.

\section{Effektives MRSA-Management}

Ein wirksames Management von Infektionen durch multiresistenter Erreger, insbesondere durch MRSA, setzt sich aus verschiedenen Komponenten zusammen:

- Primäres Screening

- Krankenhaushygienische Maßnahmen

- Konsequente Sanierung von Infektionen

- Kommunikation und Information

- Fort- und Weiterbildung

\section{Primäres Screening bei Krankenhausaufnahme}

Wie oben dargestellt sind Screeningprogramme zur Identifizierung unerkannter MRSA-Träger bei Krankenhausaufnahme in 2-facher Hinsicht effektiv. Sie führen zur Senkung der MRSA-Infektionen und vermindern dadurch die Behandlungs- und Isolierungskosten [16].

Ein generelles MRSA Screening wird vom Robert Koch-Institut nur bei ei- ner besonders hohen MRSA-Inzidenz als sinnvoll angesehen [21].

Im Normalfall sollte ein selektives Screening bei der Krankenhausaufnahme unter Berücksichtigung verschiedener Risikofaktoren (bekannte MRSA-Anamnese, Patienten, die aus Regionen bzw. Einrichtungen mit bekannt hoher MRSAPrävalenz verlegt werden, Kontaktpatienten von MRSA-Trägern, aber auch Patienten mit chronischer Pflegebedürftigkeit, liegenden Kathetern, Dialysepflichtigkeit, Hautläsionen, chronischen Wunden und Brandverletzungen) durchgeführt werden [21]. Die Entnahme des Screeningabstrichs erfolgt üblicherweise in der Nase, wobei eine Kombination mehrerer Abstrichorte die Sensitivität erhöht (insbesondere Wunde oder Kathetereintrittsstelle) [21].

Optimalerweise sollte bis zum Vorliegen des Ergebnisses eine Isolierung des Patienten erfolgen, was jedoch häufig aus Kapazitäts- und Kostengründen nicht möglich ist [11].

\section{Krankenhaushygienische Maßnahmen}

Die wichtigsten Hygienemaßnahmen im Umgang mit multiresistenten Erregern wurden von Instituten wie dem Robert Koch-Institut festgelegt [18] und sind im Internet abrufbar ( $\bullet$ Infobox 1). Auf einige Stichpunkte soll explizit hingewiesen werden:

- Unterbringung des MRSA-Trägers im Einzelzimmer oder ggf. Kohortenisolierung

- Einhalten entsprechender Hygienemaßnahmen wie Tragen von Schutzkleidung inklusive Mund- und Nasenschutz sowie Handschuhen

- Hygienische Händedesinfektion vor und nach jedem Patientenkontakt

- Desinfektion aller Gegenstände, die vom oder am Patienten benutzt wurden

- Indikationsstellung einer Eradikationstherapie

- Dokumentation aller durchgeführten Maßnahmen und Information

\section{Konsequente Sanierung von Infektionen}

Besteht eine manifeste Infektion durch MRSA, sollte diese schnellstmöglich effektiv behandelt werden. Im Fachbereich der Unfallchirurgie und Orthopädie sollte die dies nach den üblichen Behandlungsgrundsätzten in der Therapie der akuten und chronischen Osteitis, Gelenkinfektionen und periprothetischen Infektionen durchgeführt werden. Im Fall eines komplizierten Behandlungsverlaufs sollte die Verlegung in ein Zentrum mit Erfahrung in der „septischen Chirurgie“ erwogen werden.

\section{Kommunikation und Information}

Die Isolierungsmaßnahmen bedeuten für den betroffenen Patienten erhebliche Einschränkungen und Unannehmlichkeiten. Sie führen zur Verunsicherung des Patienten und seiner Angehörigen und werden häufig als „diskriminierend“ empfunden. Daher müssen alle Maßnahmen dem Patienten selbstverständlich ausführlich erklärt und auch seine Angehörigen aufgeklärt und informiert werden.

Bei Transport, Verlegung oder Entlassung von MRSA-Patienten sollten entsprechende Einrichtungen oder der Hausarzt über die Besiedelung oder Infektion informiert werden, um ggf. Schutzmaßnahmen treffen zu können [11].

\section{Fort- und Weiterbildung}

Damit die Screening- und Hygienemaßnahmen reibungslos durchgeführt werden können, sind Schulungen aller an der Patientenversorgung beteiligter Berufsgruppen notwendig. Nur so können eine Verunsicherung über die Verbreitung von und die Gefährdung durch MRSA vermieden und eine motivierte Mitarbeit ermöglicht werden.

\section{Fazit}

Die aktuelle Prävalenz methicillinresistenter Staphylococcus-aureus-Stämme beträgt in Deutschland 21\%. In Kombination mit einer zunehmenden MRSAZirkulation zwischen verschiedenen Einrichtungen des Gesundheitssystems, ei- 
ner erschwerten effektiven Therapie von manifesten MRSA-Infektionen und knapper werdenden Ressourcen im Gesundheitssystem stellt dies eine kritische Situation dar. Nachdem in den letzten Jahren die Risikofaktoren für eine MRSA-Besiedelung und Übertragung erkannt worden sind, muss nun ein effektives MRSAManagement zur Bekämpfung und Eindämmung der resistenten Erreger eingesetzt werden. Eckpunkte desselben sind: - primäres selektives MRSA-Screening,

- krankenhaushygienische Maßnahmen,

- konsequente chirurgische Sanierung von Infektionen,

- Information von Patienten, Angehörigen und weiterbehandelnden Einrichtungen und

- Fort- und Weiterbildung aller an der Patientenversorgung beteiligten $\mathrm{Be}$ rufsgruppen.

\section{Korrespondenzadresse}

\section{Dr. M. Diefenbeck}

Klinik für Unfall-, Hand-

und Wiederherstellungschirurgie,

Friedrich-Schiller-Universität Jena,

Erlanger Allee 101, 07747 Jena

Michael.Diefenbeck@med.uni-jena.de

Interessenkonflikt. Der korrespondierende Autor gibt an, dass kein Interessenkonflikt besteht.

\section{Literatur}

1. Al-Nammari SS, Bobak P, Venkatesh R (2007) Methicillin-resistant Staphylococcus aureus versus Methicillin-sensitive Staphylococcus aureus adult haematogenous septic arthritis. Arch Orthop Trauma Surg 127: 357-362

2. Davis KA, Stewart JJ, Crouch HK et al. (2004) Methicillin-resistant Staphylococcus aureus (MRSA) nares colonization at hospital admission and its effect on subsequent MRSA infection. Clin Infect Dis 39: 776-782

3. Elek SD, Conen PE (1957) The virulence of Staphylococcus pyogenes for man; a study of the problems of wound infection. Br J Exp Pathol 38: 139144

4. European Antimicrobial Resistance Surveillance System (EARSS) (2005) Anual report. EARSS, Bilthoven

5. Facklam R, Hollis D, Collins MD (1989) Identification of gram-positive coccal and coccobacillary vancomycin-resistant bacteria. J Clin Microbiol 27: 724-730

6. Geldner G, Ruoff M, Hoffmann HJ et al. (1999) [Cost analysis concerning MRSA-infection in ICU]. Anasthesiol Intensivmed Notfallmed Schmerzther 34: 409-413
7. Gristina AG, Oga M, Webb LX et al. (1985) Adherent bacterial colonization in the pathogenesis of osteomyelitis. Science 228: 990-993

8. Hayes PD, Nasim A, London NJ et al. (1999) In situ replacement of infected aortic grafts with rifampicin-bonded prostheses: The Leichester experience (1992 to 1998). J Vasc Surg 30: 92-98

9. Henrichfreise B, Wiegand I, Pfister W et al. (2007) Resistance mechanisms of multiresistant Pseudomonas aeruginosa from Germany and correlation with hypermutation. Antimicrob Agents Chemother 51: 4062-4070

10. Herr CE, Heckrodt TH, Hofmann FA et al. (2003) Additional costs for preventing the spread of methicillin-resistant Staphylococcus aureus and a strategy for reducing these costs on a surgical ward. Infect Control Hosp Epidemiol 24: 673-678

11. Hornberg C, Knoop D, Kipp F (2006) Bedeutung von MRSA in der Patientenversorgung. Orthopäde 35: 1159-1168

12. Kilgus DJ, Howe DJ, Strang A (2002) Results of periprosthetic hip and knee infections caused by resistant bacteria. Clin Orthop Relat Res 404: 116124

13. Kipp F, Friedrich A, Becker K et al. (2005) Bedrohliche Zunahme Methicillin-resistenter Staphylococcus-aureus-Stämme. Dtsch Ärztebl A-102: 2045-2050

14. Kreuzer J, Nußbaum B, Bernd L et al. (2002) Reale Kosten der MRSA-Osteitis - eine retrospektive Studie im Zeitraum von 1996-2001. Trauma Berufskrankh 4: 344

15. Murphy GJ, Pararajasingam R, Nasim A et al. (2001) Methicillin-resistant Staphylococcus aureus infection in vascular surgical patients. Ann R Coll Surg Engl 83: 158-163

16. Nixon M, Jackson B, Varghese P et al. (2006) Methicillin-resistant Staphylococcus aureus on orthopaedic wards. J Bone Joint Surg Br 88-B: 812-817

17. Popp W, Hilgenhörner $M$, Leisebein T et al. (2003)Personalkosten durch Isolierungsmaßnahmen von MRSA-Patienten. Gesundheitsökonom Qualitätsmanag 8: 187-190

18. Robert Koch-Institut (1999) Empfehlung zur Prävention und Kontrolle von Methicillin-resistenten Staphylococcus aureus-Stämmen (MRSA) in Krankenhäusern und anderen medizinischen Einrichtungen. Bundesgesundheitsbl Gesundheitsforsch Gesundheitsschutz 42: 954-958

19. Robert-Koch-Institut (2003) Methicillin-resistenter Staphylococcus aureus (MRSA) in deutschen Alten- und Pflegeheimen - zur Situation. Epidemiol Bul 19

20. Robert Koch-Institut (2004) Zur MRSA-Situation in Deutschland im Jahr 2003. Epidemiol Bull 42

21. Robert Koch-Institut (2005) Zum Management des MRSA-Screenings. Epidemiol Bull RKI 42: 385-391

22. Whitby M, McLaws ML, Berry G (2001) Risk of death from methicillin-resistant Staphylococcus aureus bacteraemia: a meta-analysis. Med J Aust 175: 264-267 